\title{
Radiation chemistry approach to the study of ice analogs
}

\section{Maria Colin-Garcia, Alicia Negrón-Mendoza, and Sergio Ramos-Bernal}

\author{
Instituto de Ciencias Nucleares, Universidad Nacional Autónoma de México, \\ Circuito Exterior, Cd. Universitaria, 04510 México, D. F., México \\ email: negron@nucleares.unam.mx
}

\begin{abstract}
The aim of this work is to study the chemistry of the irradiation of frozen solutions of HCN. This compound has been detected in comets and other icy bodies. The CN group might have made its first appearance in the early stages of chemical evolution. Therefore, is behavior under irradiation at low temperature is relevant for chemical evolution studies and icy bodies.
\end{abstract}

Keywords. Astrobiology, astrochemistry, molecular processes, laboratory, comets

\section{Introduction}

In the light of the present knowledge of ices in the Solar System, a radiation chemistry approach can be very useful to study the behavior of compounds connected not only to these bodies, but also to interstellar chemistry.

Comets are thought to be carriers of material that was initially available on Earth. The Deep Impact mission to Comet $9 \mathrm{P} /$ Tempel 1 showed the presence of $\mathrm{H}_{2} \mathrm{O}, \mathrm{C}_{2} \mathrm{H}_{6}$, $\mathrm{C}_{2} \mathrm{H}_{6}, \mathrm{HCN}, \mathrm{CO}, \mathrm{CH}_{3} \mathrm{OH}, \mathrm{H}_{2} \mathrm{CO}, \mathrm{C}_{2} \mathrm{H}_{2}$, and $\mathrm{CH}_{4}$ (Mumma et al. 2005). To this end, the organic inventory is of central importance concerning the origin of life.

The comet is exposed to radiation for more than 4.6 billion years. The amount of energy deposited in a cometary nucleus comes from internal and external sources (Whipple 1977).

In simulation experiments it is possible to use radiation sources which are more common in radiation chemistry laboratories, with the same LET as that protons at cosmic energies. The gamma radiation of ${ }^{60} \mathrm{Co}$ has an average LET of $0.23 \mathrm{KeV}$, similar to that of billon electron-volt protons. This means that the average energy deposit along the radiation paths, and so the distribution of reactive species and their reactions, is similar (Draganic et al. 1984).

\section{Experimental}

Hydrogen cyanide was generated in a special setup from KCN and sulfuric acid. The concentrations ranged from 0.001 to 0.2 moles $\mathrm{dm}^{-3}$ and the $\mathrm{pH}$ was $6-9$. The frozen $(77 \mathrm{~K})$ samples were prepared in an argon atmosphere. The irradiation was carried out using a ${ }^{60}$ Co source. The sample radiation doses were from $3 \mathrm{kGy}$ to $419 \mathrm{kGy}$. After irradiation, the samples were thawed at room temperature. The analysis was made according to the procedures followed by Colin et al. (2008). 


\section{Results and discussion}

The inventory of products include several types of compounds such as gases and non volatile products that remain in the solution. Among the gases are carbon monoxide, carbon dioxide, methane, ammonia, and hydrogen. To analyze non-volatile products, the solution was lyophilized and the dry residue hydrolyzed and analyzed for small molecules, to reveal the formation of biologically important compounds. The amounts and aspect of the dry residue depend on the absorbed dose. The infrared spectra of the dry residue showed characteristic absorption bands that suggest that their constituents are mainly polyamides, urea, and substituted urea.

The decomposition of HCN formed about $76 \%$ of the oligomeric material. This product constituted the main component of the dry residue. The estimated molecular weight for such oligomers is up to 20000 daltons. The formation of the oligomers was observed in all the concentrations and doses studied. Oligomer concentration increased with the dose, and after the depletion of cyanide the build up ended. It was found that cyanide solutions of $10^{-3}$ moles $\mathrm{dm}^{-3}$ irradiated at $2.5 \mathrm{kGy}$, at room temperature, are enough to produce these types of compounds.

\section{Final Remarks}

The chemical action of ionizing radiation consists essentially in producing chemically reactive species (free radicals, radical-ions). In water-dominated, CN-containing mixtures, radicals from water ices react by addition of abstraction with CN molecules and produce oligomers. The data obtained suggest that they are formed by polyamides and esters. Upon hydrolysis, these oligomers released compounds of biological significance like amino acids, purines and carboxylic acids. These oligomeric materials present the same characteristics in the frozen and in the liquid multi-component systems. The yields in ice were one order of magnitude lower. This is mainly due to the rigid structure of the ice and the limited mobility of the radicals at low temperature. The results of this study underline the importance of radiation-induced reactions as an energy source in extraterrestrial scenarios like in comets or other icy objects.

\section{Acknowledgements}

This work was supported by DGAPA-UNAM Grant IN223406. The support of the Posgrado en Ciencias Biológicas, UNAM for one of us (MC) is acknowledged.

\section{References}

Colín-García, M., Negrón-Mendoza, A., \& Ramos-Bernal, S. 2008. Astrobiology, in press Draganič, I. G., Draganič, S. D., \& Vujosevič, S. 1984, Icarus, 60, 464

Mumma, M. J., Di Santi, M. A., Magee-Sauer, K., Bonev, B. P., Villanueva, G. L., Kawakita, H., Dello Russo, N., Gibb, E. L., Blake, G. A., Lyke, J. E., Campbell, R. D., Aycock, J., Conrad, A., \& Hill, G. M. 2005, Science, 310, 270

Whipple, P. L. 1977, in: A. H. Delsemme (ed.), Comets, asteroids, meteorites, University of Toledo, (Toledo) p. 25 